\title{
Guided Ultrasonic Waves for Impact Damage Detection in Composite Panels
}

\author{
B.I.S. Murat, P. Khalili and P. Fromme \\ Department of Mechanical Engineering, University College London, WC1E 7JE, UK
}

\begin{abstract}
Carbon fiber laminate composites, consisting of layers of polymer matrix reinforced with high strength carbon fibers, are increasingly employed for aerospace structures. They offer advantages for aerospace applications, e.g., good strength to weight ratio. However, impact during the operation and servicing of the aircraft can lead to barely visible and difficult to detect damage. Depending on the severity of the impact, fiber and matrix breakage or delaminations can occur, reducing the load carrying capacity of the structure. Efficient structural health monitoring of composite panels can be achieved using guided ultrasonic waves propagating along the structure. Impact damage was induced in the composite panels using standard drop weight procedures. The guided wave scattering at the impact damage was measured using a noncontact laser interferometer, quantified, and compared to baseline measurements on undamaged composite panels. Significant scattering of the first anti-symmetrical $\left(\mathrm{A}_{0}\right)$ guided wave mode was observed, allowing for the detection of barely visible impact damage. The guided wave scattering was modeled using full three-dimensional Finite Element (FE) simulations, and the influence of the different damage mechanisms investigated. Good agreement between experiments and predictions was found. The sensitivity of guided waves for the detection of barely visible impact damage in composite panels has been verified.
\end{abstract}

Keywords: Guided Ultrasonic Waves, Impact Damage, Composites

\section{INTRODUCTION}

The usage of composite materials in aerospace structures has increased significantly as they offer significant advantages such as an improved strength to weight capacity. However, the combination of carbon fibers and epoxy matrix in typical carbon-fiber reinforced polymer (CFRP) prepreg composites is susceptible to impact loading. Low-velocity impact loading can induce barely visible damage [1], including matrix cracking, delaminations, and fiber breakage, that can reduce the integrity and load bearing capacity of the structure. Defects in the structure can potentially result in a catastrophic failure if exposed to repetitive loading [2]. Therefore, it is important to efficiently monitor the composite structure during its service life to detect such damage and to ensure the safe operation of the structure $[3,4]$.

Guided ultrasonic waves have the potential for the efficient monitoring of large structures, as they can propagate over considerable distances at low excitation frequencies. This could significantly reduce the inspection time for large structures and be employed as part of a structural health monitoring system (SHM) [5, 6]. Guided waves have been studied and applied for the non-destructive evaluation (NDE) of composite structures using contact or air coupled transducers for the generation and sensing of the guided waves. The capacity for the detection of delaminations [7, 8], cracks [6,9], and for material characterization [10] has been shown. Guo and Cawley [7] demonstrated the detection of delaminations in CFRP laminates from reflected waves. Toyama et al. [11] investigated the interaction of the first symmetric Lamb wave mode $\left(\mathrm{S}_{0}\right)$ with cracks and delaminations on cross-ply CFRP plates. Ramadas et al. [12] studied the first antisymmetric wave mode $\left(\mathrm{A}_{0}\right)$ mode propagation and the mode conversion at delaminations. However, due to the complexity of the guided wave propagation in composites, the practical testing needs to be optimized for the specific application. Many factors can affect the propagation and scattering of the guided waves [13], i.e., transducer effects [14], the properties of the composite structure [15], as well as parameters of the impact damage [9,16]. Finite element analysis (FEA) can be used to investigate the complex scattering behavior of guided ultrasonic waves at impact damage and improve the understanding of the interaction and potential for damage characterization [17]. Previous studies have shown that it can be challenging to accurately model impact damage in a laminated composite plate $[18,19]$.

Health Monitoring of Structural and Biological Systems 2014, edited by Tribikram Kundu, Proc. of SPIE Vol. 9064,906419 .

(C) 2014 SPIE · CCC code: 0277-786X/14/\$18 · doi: 10.1117/12.2046309 


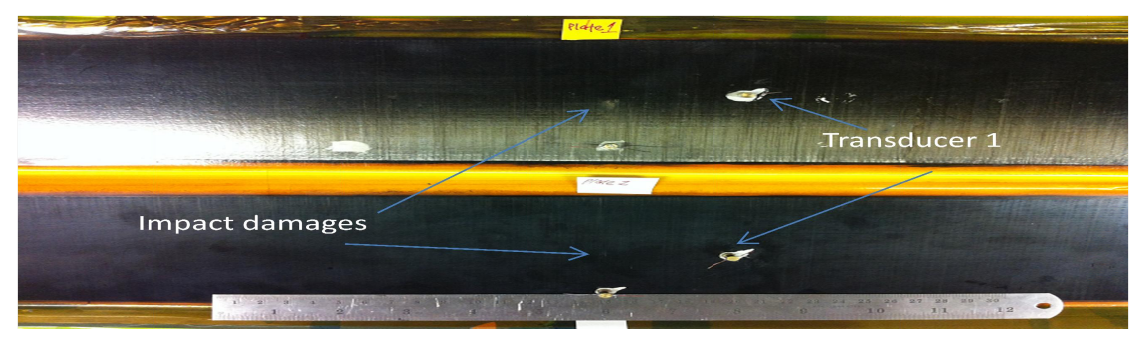

Figure 1: Composite plate specimens with impact damage and transducer locations shown.

This is due to the occurrence of multiple damage modes in a plate with impact damage, such as delaminations, cracks and fiber breakages. In this contribution the propagation and the scattering of the $\mathrm{A}_{0}$ guided ultrasonic wave mode has been investigated using a 3D FEA model [20] of a composite plate containing a damaged area consisting of a delamination and changed material properties. The guided wave scattering at the damage area has been compared to experimental results obtained for composite plates with barely visible impact damage [21].

\section{EXPERIMENTAL METHODOLOGY}

Two specimens (Fig. 1) were supplied by the Composite Systems Innovation Centre, University of Sheffield, which had been investigated in a separate study [1]. The composite plates $(990 \mathrm{~mm} \times 110 \mathrm{~mm} \times 2 \mathrm{~mm})$ were fabricated with unidirectional prepregs by autoclave cure using Cytec 977-2 / Tenax HTS cross-ply laminates. The plates consist of 8 prepreg layers with a symmetric layup sequence of $[0 / 90]_{2 s}$. Additionally, the plates contain a $25 \mu \mathrm{m}$ thick polymide film and an $18 \mu \mathrm{m}$ thick layer of flexible printed circuit boards for electrical resistance measurements as detailed in [1]. The specimens were subjected to a $7.4 \mathrm{~J}$ impact damage using a hemispherical $15 \mathrm{~mm}$ impactor head and following standard drop weight impact procedures. A small degree of fiber fracture and indentation is visible on the surface of the plates.

A piezoelectric transducer consisting of a piezoelectric disc (Ferroperm Pz27, $5 \mathrm{~mm}$ diameter, $2 \mathrm{~mm}$ thickness) and a brass backing mass $(5 \mathrm{~mm}$ diameter, $6 \mathrm{~mm}$ height) was glued onto the plate with Loctite 2-part epoxy approximately $50 \mathrm{~mm}$ from the center of the impact damage. The $\mathrm{A}_{0}$ guided wave mode was excited by the transducer as a 5 cycle sinusoidal wave packet with a center frequency of $100 \mathrm{kHz}$. The excitation signal was generated in a programmable function generator and amplified using a power amplifier. The out-of-plane velocity was measured using a laser vibrometer fixed to a scanning rig and moved parallel to the specimen. The time traces of the received signals were filtered using a bandpass filter (cut-off frequencies set as $\pm 25 \%$ of the pulse center frequency) and were recorded and averaged (20 averages) using a digital oscilloscope. All signals were then saved to a PC and were further analyzed using Matlab. The signals were digitally filtered using a Butterworth band pass filter $\left(4^{\text {th }}\right.$ order, $\pm 50 \%$ of center frequency). The maxima of the signal envelopes obtained using Hilbert transform were evaluated.

Two types of scans were used; (i) horizontal line scans over a length of $200 \mathrm{~mm}$ from the transducer location in both directions along the specimen with $1 \mathrm{~mm}$ step size; and (ii) circular scans with $30 \mathrm{~mm}$ radius measured every $5^{\circ}$ around the excitation location, impact damage and a symmetrically located undamaged area. Measurements on the undamaged part of the specimens were performed as a baseline measurement and to study the wave propagation characteristics of the $\mathrm{A}_{0}$ Lamb wave mode in the composite plates. Figure 2 illustrates the geometry of the composite plate specimens, with the impact damage approximately in the center, as well as the laser measurement locations.

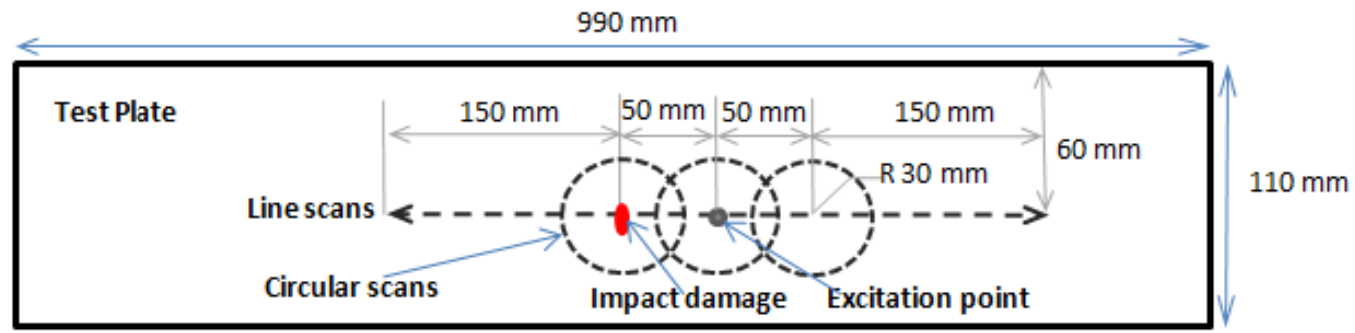

Figure 2: Composite plate specimen geometry and laser measurement locations. 


\section{FINITE ELEMENT MODELING}

In order to investigate the guided wave propagation and scattering in a composite structure with impact damage a full three-dimensional (3D) FE model of a large composite plate of similar properties to the specimen described above was developed, as shown in Fig 3. A plate with dimensions of $1 \mathrm{~m} \times 1 \mathrm{~m} \times 2 \mathrm{~mm}$ was modeled using MATLAB input codes. The composite plate consists of 4 layers through the thickness; the plate was treated as an orthotropic, homogenous structure. The material properties of the plate were set as a bi-directional composite obtained from a reference [22], with Rayleigh damping (damping ratio $\beta=60 \mathrm{~ns}$ ) to obtain the material damping presented as the imaginary part of stiffness values. Element size of $1 \mathrm{~mm}$ in both the $\mathrm{x}$ - and $\mathrm{y}$ - direction and $0.5 \mathrm{~mm}$ in the $\mathrm{z}$-direction (thickness) were employed. This mesh size resulted in the generation of 4 million elements throughout the plate. The element type was chosen as an 8 -node linear brick with reduced integration (C3D8R). Numerical convergence of the simulations was investigated and the element size as $1 / 14^{\text {th }}$ of the wavelength $(\lambda=14 \mathrm{~mm})$ was found to be sufficiently small to accurately model the guided wave propagation.

Uniform out-of plane excitation in the middle of the thickness of the model was introduced to generate an $\mathrm{A}_{0}$ Lamb wave propagating in the plate. The excitation consisted of a 5 cycle sinusoidal tone burst modulated by a Hanning window with $100 \mathrm{kHz}$ center frequency. This achieves a reasonable compromise between time duration of the pulse to separate different pulses and frequency bandwidth, which in turn influences the necessary element size to fulfill the stability criteria relative to the wavelength. The out-of-plane displacement was monitored at different locations in the plate to match the experimental measurements. The time trace at each monitoring node was time gated to remove edge reflections from the plate boundaries and the maximum amplitude extracted similar to the experiments. Initial simulations were preformed for an undamaged composite plate. A line scan along the composite fiber direction was carried out to study the amplitude decay of the signal as it propagates in the plate. A circular scan around the excitation point at $30 \mathrm{~mm}$ radius was obtained to investigate the amplitude variation in different propagation directions.

The impact damage was model as a rectangular shape with zero-volume to create a symmetric delamination with a size of $20 \mathrm{~mm} \times 20 \mathrm{~mm}$. A layer of elements attached to the delaminated surface was assigned reduced stiffness values. The real parts of the material stiffness values were reduced by $75 \%$ as a simple model of the multi-mode impact damage (e.g., fiber breakage and matrix cracking). The excitation point was placed $50 \mathrm{~mm}$ from the center of the delamination in the $45^{\circ}$ direction (relative to the delamination orientation). This helped to eliminate an energy focusing effect caused by the parallel edges of the delamination. A circular scan of $30 \mathrm{~mm}$ radius was conducted to study the scattering pattern around the defective area. The same simulation was performed for a defect free plate to obtain baseline data.

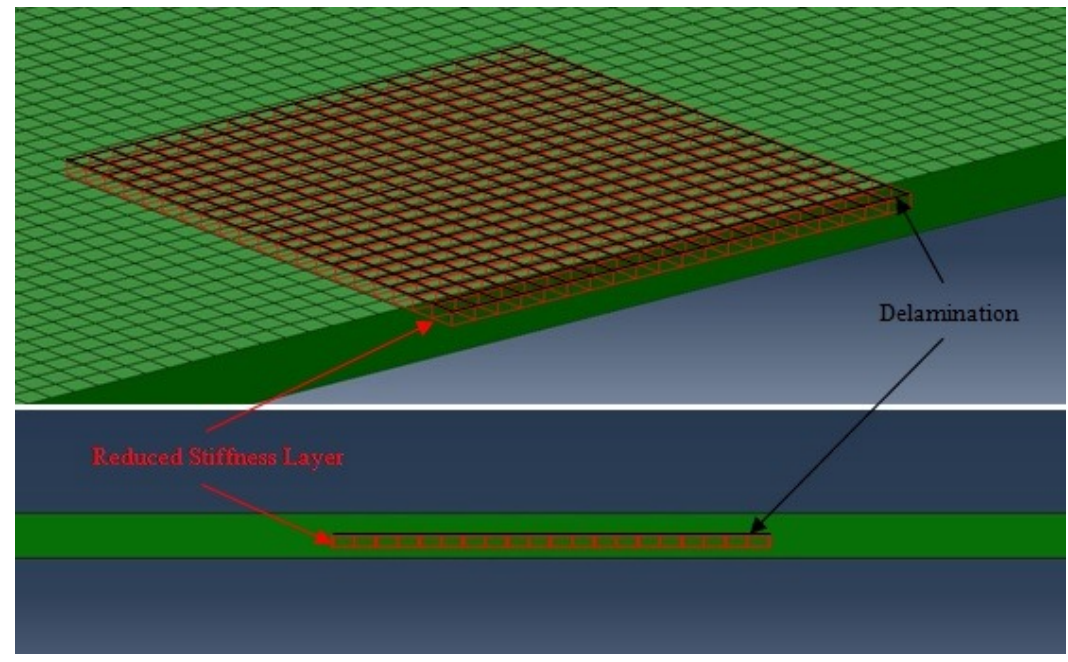

Figure 3: FE model of composite plate with symmetric delamination, reduced stiffness layer, and mesh shown. 


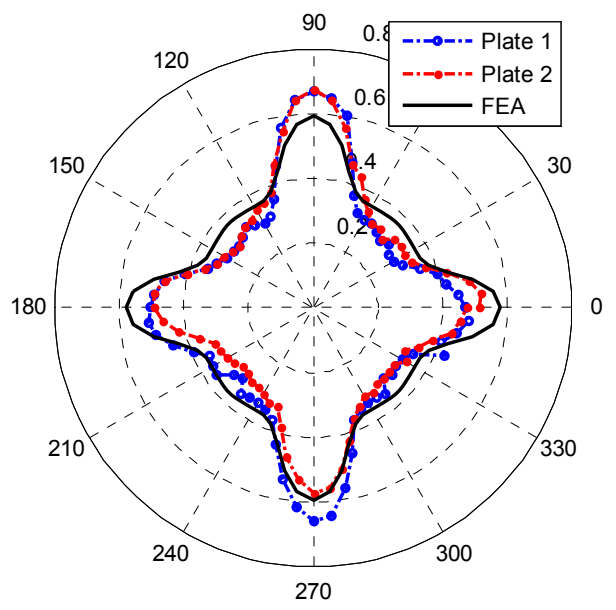

Figure 4: Polar plot of excitation amplitude variation with angular direction around transducer; excitation frequency $100 \mathrm{kHz}$; measured every $5^{\circ}$ at $30 \mathrm{~mm}$ radius; maximum amplitude of signal envelope (Hilbert transform).

\section{GUIDED WAVE PROPAGATION IN COMPOSITE PANELS}

In order to optimize the guided wave testing for damage detection, it is important to have a clear understanding of the guided wave propagation characteristics in the composite specimens. This section reports the characteristics of the $\mathrm{A}_{0}$ guided wave mode excitation and propagation measured in the undamaged areas of the plates. Fig. 4 shows the polar plot of the excitation amplitude variation with angular direction around the piezoelectric transducer. The piezoelectric transducer acts in good approximation as a point source, but for an anisotropic composite plate a directionality pattern of the excited guided wave mode is expected [23]. It can be seen in Fig. 4 that the amplitudes of the guided wave mode are higher in the $0^{\circ}$ and $90^{\circ}$ direction (along the fiber orientation) than in other directions, with the $90^{\circ}$ direction signal amplitude almost double the value as compared to the $45^{\circ}$ direction. The excitation amplitude and pattern was very repeatable between the two composite panels. For the FE simulation result, a consistent level of amplitude concentration can be observed as the FE model uses homogenized material properties. The experimental results show slightly higher amplitude in the $90^{\circ}$ direction compared to the $0^{\circ}$ direction. The symmetric outer layers of the composite plates have the fibers arranged in the $90^{\circ}$ direction, thus having higher bending stiffness in this direction. Overall, a repeatable amplitude variation from the experimental results for both plates can be observed which is consistent with the FE simulation.

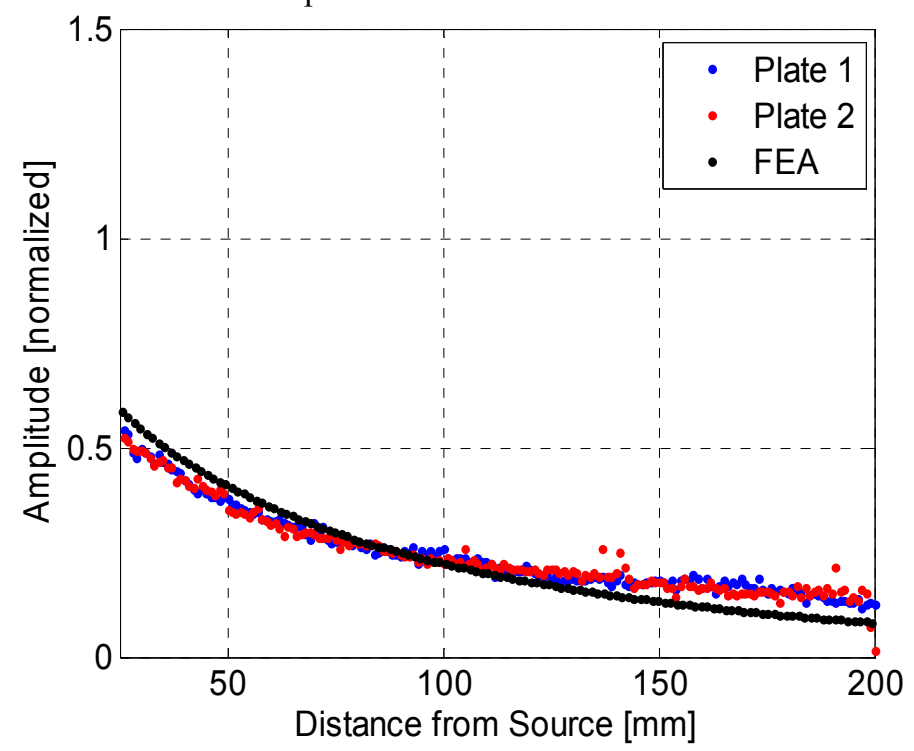

Figure 5: Amplitude of propagating guided wave pulse in intact composite plate; excitation frequency $100 \mathrm{kHz}$; measured every 1 mm step; maximum amplitude of signal envelope (Hilbert transform). 
Fig. 5 shows the amplitude of the guided wave pulse propagating in the intact part of the two composite plates together with the FEA prediction. The guided wave propagation was monitored in $1 \mathrm{~mm}$ steps along a $200 \mathrm{~mm}$ line in the $0^{\circ}$ direction. For each measurement point the maximum amplitude of the wave pulse was extracted using the Hilbert transform. The amplitude measurements on both plates give repeatable results. The experimental curves show that the decaying amplitude decrease does not directly follow the $1 / \sqrt{r}$ wave spreading. The effects of material damping and anisotropy influence the wave propagation, e.g., an additional amplitude decrease due to damping is typically observed for composite materials. Similar observations were obtained from the FEA prediction. It appears however that the amplitude reduction pattern does not match exactly, which could be related to additional factors which should be investigated further.

\section{GUIDED WAVE SCATTERING AT IMPACT DAMAGE}

The propagation and scattering of the guided waves on the area containing the impact damage area was observed. Measuring the wave propagation across the area containing the impact damage, a significant change in signal shape and increase in amplitude can be observed. Fig. 6 shows the amplitude of the propagating guided wave pulse over the damaged part of the composite plate. High amplitudes can be observed around the center of the impact damage at $50 \mathrm{~mm}$ in both plates. This indicates the presence of the damage, and matched well with the visually observed width of the impact damage. This could be due to a delamination or fiber and matrix cracking induced by the impact. Between $30 \mathrm{~mm}$ and $50 \mathrm{~mm}$ a small periodic variation of the signal amplitude can be seen, which might be due to interference between the incident wave pulse and reflections at the impact damage. Behind the damage location $(50 \mathrm{~mm})$ a significant reduction of the wave pulse can be observed as compared to Fig. 5 (measurement on undamaged plate). This might allow the detection of impact damage from monitoring the amplitude of guided wave pulses between fixed sensors on a structure, as the amplitude reduction is similar for the two plate specimens with impact damage. From Fig. 6, it can be seen that the FEA provided a good prediction of the experimental observations with high amplitudes observed around the damage area and showing a reduction in amplitude behind the damage area.

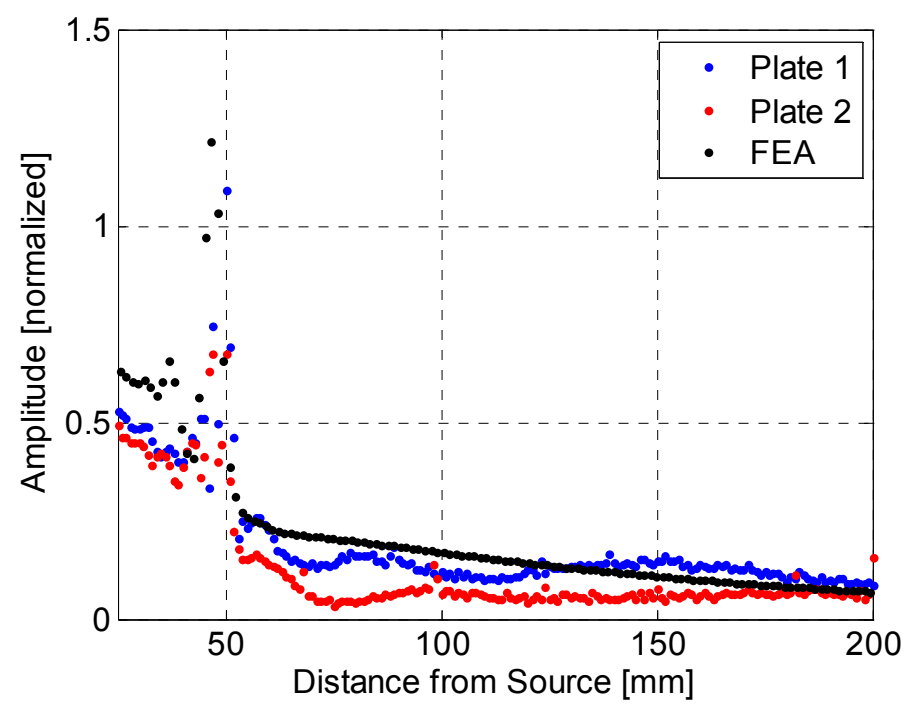

Figure 6: Amplitude of propagating guided wave pulse over damaged part of composite plate, impact damage at $50 \mathrm{~mm}$; excitation frequency $100 \mathrm{kHz}$; measured every $1 \mathrm{~mm}$ step; maximum amplitude of signal envelope (Hilbert transform). 

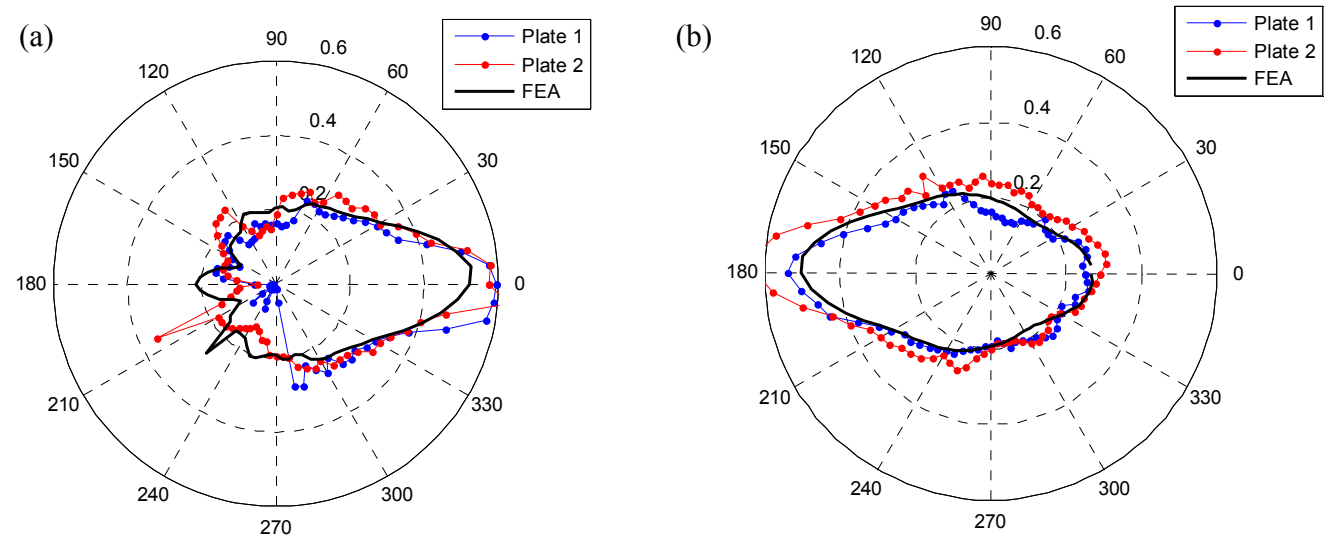

Figure 7: a) Polar plot of amplitude variation with angular direction around impact damage; b) baseline measurement on undamaged plate, symmetric to damage location; excitation frequency $100 \mathrm{kHz}$; measured every $5^{\circ}$ at $30 \mathrm{~mm}$ radius; maximum amplitude of signal envelope (Hilbert transform).

In order to understand the wave propagation and scattering around the defect, a circular scan was performed on each composite plate around the impact damage location (Fig. 7a). Another circular scan was performed in the symmetric location on the undamaged area as baseline data, shown in Fig. 7b. For the baseline measurement, one can observe a good agreement between the measurements on the two plates. The amplitude in the incident wave direction $\left(180^{\circ}\right)$ is about twice the amplitude in the $0^{\circ}$ direction, similar to the amplitude decrease observed in Fig. 6 . The amplitudes in the $90^{\circ}$ and $270^{\circ}$ directions are significantly lower than what would be expected in an isotropic metallic plate due to the energy concentration along the fiber directions (shown in Fig. 4), leading to an oval shaped amplitude distribution. For the measurement around the damage location (Fig. 7a) the incident wave $\left(0^{\circ}\right.$ direction) has a similar amplitude distribution as the baseline data $\left(180^{\circ}\right.$ direction) and no significant back-scattered energy is observed. However, the amplitude of the guided wave propagating past the impact damage is significantly reduced for both specimens. Especially for plate 2 scattering approximately $45^{\circ}$ relative to the wave propagation direction seems to occur, but for plate 1 the scattered field is not symmetric and almost no energy is scattered between $180^{\circ}$ and $270^{\circ}$. As each impact damage is unique such variations are expected, but similarities in the scattered guided wave field for both specimens can be observed. From the FE simulations, the result from the baseline measurement (Fig. 7b) shows a comparable amplitude profile with the experimental results for both plates. The amplitudes are higher along the fiber directions due to the larger stiffness. For the impact damage, the FE simulations predicted a decrease in the amplitudes behind the damage position $\left(180^{\circ}\right)$, but by a smaller amount than observed experimentally. This is in agreement with the amplitudes observed in the line scan at $80 \mathrm{~mm}$ (Fig. 6). The scattered wave from the FE simulation provides a regular pattern compared to the experimental results. This is related to the definition of the impact damage in the FE simulation as a rectangular delamination with a layer of decreased stiffness, rather than the actual complex, multimode impact damage. However, in general the FE prediction matches reasonably well with the experimental results.

\section{CONCLUSIONS}

The use of the $A_{0}$ guided ultrasonic wave mode for the detection of impact damage on composite panels has been investigated using experiments and FE simulations. From measurements on the undamaged composite plate structure, the guided wave propagation patterns were quantified. Good agreement between the experimental results and FE predictions using homogenized material properties was obtained. Scattering of the guided wave at the impact damage was observed experimentally, with increased amplitude at the impact location, and a repeatable scattering pattern with significant amplitude reduction of the guided wave propagating past the damage location. Using a regular damage implementation in the FE simulations as a square delamination with a layer of reduced stiffness, a reasonable match could be observed. The potential for the detection of impact damage in composite structures has been demonstrated, but the interaction of the guided waves with the impact damage requires further investigation. 


\section{ACKNOWLEDGEMENTS}

Bibi Intan Suraya Murat gratefully acknowledges funding by Ministry of Science, Technology and Innovation (MOSTI), Malaysia and Universiti Teknologi MARA (UiTM), Malaysia. The authors would like to thank the Composite Systems Innovation Centre, University of Sheffield, UK for the provision of the composite plates.

\section{REFERENCES}

[1] Swait, T. J., Jones, F. R. and Hayes, S. A., "A practical structural health monitoring system for carbon fibre reinforced composite based on electrical resistance," Compos. Sci. Technol. 72, 1515-1523 (2012).

[2] Cantwell, W.J. and Morton, J., "Geometrical effects in the low velocity impact response of CFRP," Compos. Struct. 12, 39-59 (1989).

[3] Takeda, N., Sierakowski, R.L. and Malvern, L. E., "Microscopic observations of cross sections of impacted composite laminate," Compos. Technol. Rev. 4(2), 40-44 (1982).

[4] Richardson, M.O.W. and Wisheart, M.J., "Review of low impact velocity properties of composite materials," Compos. A. 27(12), 1123-1131 (1996).

[5] Rose, J. L., "Ultrasonic guided waves in structural health monitoring," Key Eng. Mater. 270, 14-21 (2004).

[6] Toyama, N., Noda, J. and Okabe, T., "Quantitative damage detection in cross-ply laminates using Lamb wave method," Compos. Sci. and Technol. 63, 1473-1479 (2003).

[7] Guo, N. and Cawley, P., "The interaction of Lamb waves with delaminations in composite laminates," J. Acoust. Soc. Am. 94(4), 2240-2246 (1993)

[8] Tan, K.S., Guo, N. and Wond, B.S., "Experimental evaluation of delaminations in composite plates by the use of Lamb waves," Compos. Sci. Technol. 53(1), 77-84 (1995).

[9] Castaings, M., Singh, D. and Viot, P., "Sizing of impact damages in composite materials using ultrasonic guided waves," NDT\&E Int. 46, 22-31 (2012).

[10] Djordjevic, B. B., "Ultrasonic characterization of advanced composite materials," Proc. of the 10th International Conference of the Slovenian Society for Non- Destructive Testing, Ljubljana, Slovenia, 47-57 (2009).

[11] Toyama, N. and Takatsubo, J., "Lamb wave method for quick inspection of impact-induced delamination in composite laminates," Compos. Sci. Technol. 64, 1293-1300 (2003).

[12] Ramadas, C., Janardhan, M. P., Balasubramaniam, K., Makarand, J. and Krishnamurthy, C. V., "Delamination size detection using time of flight of Anti-symmetric $\left(\mathrm{A}_{0}\right)$ and mode converted $\mathrm{A}_{0}$ mode of guided Lamb waves," J. Intell. Mater. Syst. Struct. 21, 817-825 (2010).

[13] Lowe, M.J.S, Neau, G. and Deschamps, M., "Properties of guided waves in composite plates, and implications for NDE," Proc. AIP 700, 214-221(2004).

[14] Konstantin, J.S. and Axel, S.H., "On attenuation and measurement of Lamb waves in viscoelastic composites," Compos. Struct. 94, 177-185 (2011).

[15] Wang, L. and Yuan, F., "Group velocity and characteristic wave curves of Lamb waves in composites: Modeling and experiments," Compos. Sci. Technol. 67(7-8), 1370-1384 (2007).

[16] Evgeny, G., Natalia, G., Artern, E., Rolf, L. and Mirko, N., "Frequency dependent directivity of guided waves excited by circular transducer in anisotropic composite plates," J. Acoust. Soc. Am. 132 (2), 119-124 (2012).

${ }^{[17]} \mathrm{Ng}$, C.T., Veidt, M., Rose, L.R.F. and Wang, C.H., "Analytical and finite element prediction of Lamb wave scattering at delaminations in quasi-isotropic composite laminates," J. Sound Vibr, 331, 4870-4883(2012).

[18] Feng, D. and Aymerich, F., "Finite element modeling of damage induced by low-velocity impact on composite laminates," Compos. Struct. 108, 161-171 (2014).

[19] Perillo, G., Vedivik, N. and Echtermeyer, A., "Damage development in stitch bonded GFRP composite plates under low velocity impact: Experimental and numerical results," J. Compos. Mater. 0, 1-15 (2014).

[20] Murat, B.I.S., Khalili, P. and Fromme, P., "Impact damage detection in composite panels using guided ultrasonic waves," AIP Conf. Proc. 1581, 286-293 (2014).

[21] Murat, B.I.S. and Fromme, P., "Detection of impact damage in composite panels using guided ultrasonic waves," Proc. SPIE 8695, 869506 (2013).

[22] Neau, G, Lowe, M.J.S. and Deschamps, M, "Propagation of Lamb waves in anisotropic and absorbing plates: theoretical derivation and experiments," AIP Conf. Proc. 615, 1062-1069 (2002).

[23] Pierce, S.G., Culshaw, B., Philip, W.R., Lecuyer, F. and Farlow, R., "Broadband Lamb wave measurements in aluminum and carbon glass fibre reinforced composite materials using non-contacting laser generation and detection," Ultrasonics 35(2), 105-114 (1997). 